\title{
Study on stiffness characteristic of hydro pneumatic suspension system
}

$$
\text { Peng Zhang }{ }^{1, \mathrm{a}} \text {; Yongchao } \mathrm{Li}^{1, \mathrm{~b}} \text {; Pengzhen } \mathrm{Li}^{1, \mathrm{c}}
$$

1School of Mechanical Electronic and Information Engineering, China University of Mining and Technology, Beijing, Beijing 100083, China

aemail: zhangpeng@cumtb.edu.cn, bemail: 1967333243@qq.com, cemail: 980098502@qq.com

Keywords: Hydro pneumatic suspension;Stiffness;Computer simulation

\begin{abstract}
The influence of different structural parameter on the stiffness characteristics of hydro-pneumatic suspension system has been simulated with MATLAB/Simulink software. The simulation results indicate that the hydro-pneumatic suspension system has the nonlinear stiffness characteristics. The dynamic stiffness is greater than the static stiffness in the same displacement, and the dynamic stiffness changes faster with the displacement. The stiffness of hydro-pneumatic suspension cylinder decreases with the increase of the initial air volume and the diameter of the piston rod.
\end{abstract}

\section{Introduction}

The hydro pneumatic suspension system has excellent nonlinear stiffness and damping characteristics. It can meet the engineering vehicle demand because of its good vibration reduction characteristics. And it is more and more widely used in the engineering vehicles[1,2]. It directly affect the vehicle ride comfort, handling and stability[3,4]. The influence of different structure parameters of hydro pneumatic suspension on damping and stiffness characteristics is of great significance for the reasonable design of the hydro pneumatic suspension system[5,6,7]. . This paper mainly carries on he stiffness characteristics of the hydro pneumatic suspension system.

\section{Stiffness calculation of hydro pneumatic suspension system}

Fig. 1 shows the structure of the single air chamber hydro-pneumatic suspension[8]. There are three chambers, A, B and C, which are composed of cylinder 3 and piston rod 5 . Chamber A is filled with high-pressure nitrogen gas. Chambers $B$ and $C$ are full of the hydraulic oil. While damping hole 1 and check valve 4 are located on the wall of piston rod, which make chambers $\mathrm{B}$ and $\mathrm{C}$ connected.

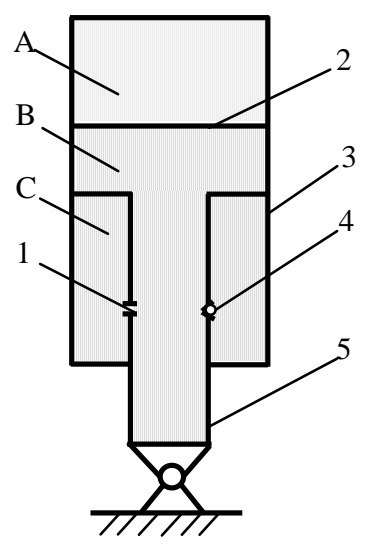

1-damping hole, 2-floating piston, 3-cylinder, 4-check valve, 5-piston rod

Fig.1. Structure of the single air chamber hydro-pneumatic suspension system

When the hydro-pneumatic suspension cylinder is under the effect of external excitation signal, it will produce the relative motion between the piston rod and cylinder. The force of the hydro-pneumatic suspension cylinder is composed of the pressure difference between chambers B and $\mathrm{C}$ and the friction between the piston rod and cylinder[9]. Because the hydraulic cylinder is in a 
state of vibration and good lubrication, the influence of friction can be ignored. Assuming that the piston rod is fixed, the excitation signal is positive upward direction. So the output force of the piston rod can be expressed as[10]:

$$
F=P_{3} A_{3}-P_{2} A_{2}
$$

Where $F$ is the force produced by the piston rod; $P_{2}$ and $A_{2}$ are the oil pressure and cross-sectional area of $\mathrm{B}$ cavity; $P_{3}$ and $A_{3}$ are the oil pressure and cross-sectional area of C cavity.

The equation of state hydro pneumatic in the suspension is:

$$
P_{1} V_{1}^{r}=P_{10} V_{10}^{r}
$$

where $P_{10}$ and $V_{10}$ are the initial pressure and volume of the gas; $P_{1}$ and $V_{1}$ are the instantaneous pressure and volume of the gas; $r$ is the polytropic constant.

$$
V_{1}=V_{10}+A_{4} * x
$$

where $A_{4}$ is the sectional area of piston rod; $x$ is the displacement of the cylinder.

While damping is not considered, there is the following relationship:

$$
P_{1}=P_{2}=P_{3}
$$

The expression of elastic force is:

$$
F_{k}(x)=F=\frac{P_{10} V_{10}^{r}\left(A_{3}-A_{2}\right)}{\left(V_{10}+A_{4} x\right)^{r}}
$$

The hydro pneumatic suspension stiffness $k(x)$ can be obtained by through the formula (5) derivation:

$$
k(x)=\frac{r P_{10} V_{10}^{r}\left(A_{2}-A_{3}\right) A_{4}}{\left(V_{10}+A_{4} x\right)^{r+1}}
$$

In the hydro pneumatic suspension system, the gas filling the suspension cylinder is as the original elastic. The volume elastic modulus of gas is much smaller than the bulk modulus of oil. So the effect of the oil compressibility on the stiffness is ignored. And the damping characteristics are also ignored in the discussion of the stiffness characteristics of the hydro pneumatic suspension. The inert gas in hydro pneumatic suspension plays a role of the elastic component. And the variable exponent of the inert gas varies with the different change of the inert gas, so the stiffness characteristic of the hydro pneumatic suspension is closely related to the variable exponent of the inert gas.

The stiffness of the hydro pneumatic suspension can be divided into static stiffness and dynamic stiffness. The stiffness of the hydro pneumatic suspension is the static stiffness when the piston rod moves slowly relative to the cylinder. And it is assumed that the inert gas is changed in an isothermal state in this process. While the stiffness of the hydro pneumatic suspension is the dynamic stiffness when the piston rod moves rapidly with respect to the cylinder. And it is assumed that the inert gas varies according to the adiabatic condition in this process.

The gas variable index is equal to 1 when it is to calculate the static stiffness of the hydro pneumatic suspension according to the formula (6). And the gas variable index is equal to 1.73 while calculating the dynamic stiffness. The stiffness characteristcs of the mining vehicle is showed as Fig.2. 


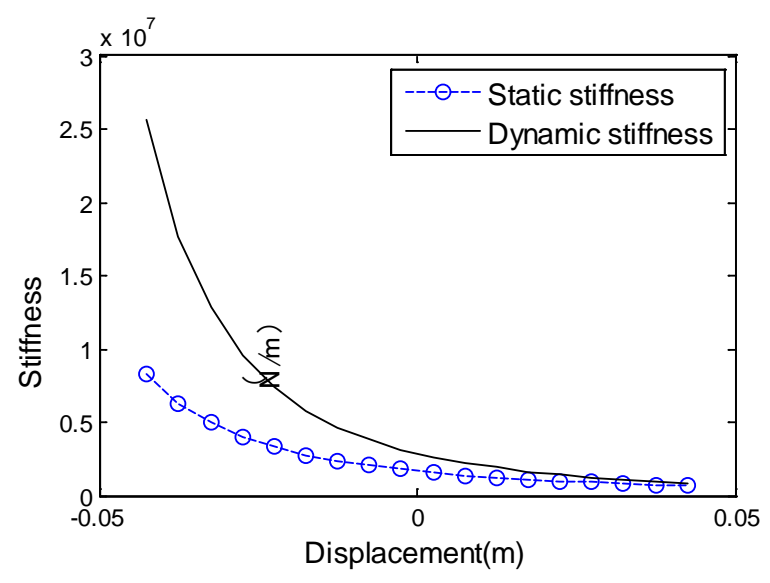

Fig. 2. Stiffness characteristics of the hydro pneumatic suspension system of the mining vehicle

As can be seen from Fig. 2, the dynamic stiffness is greater than the static stiffness in the same displacement, and the dynamic stiffness changes faster with the change of the displacement. The stiffness characteristic curve of the hydro pneumatic suspension shows the significant non-linearity. The stiffness characteristics which increases obviously with the increase of displacement can improve the ride comfort of the vehicle in various road surfaces. The input displacement of the suspension is not obvious and the change of the stiffness is not obvious when the vehicle is running on the better road, so the ride comfort of the vehicle can be better on the road. The stiffness of the suspension increases with the increase of the displacement while the vehicle runs on the poor road. And the vehicle has great anti impact ability while driving in poor pavement to ensure the ride comfort of vehicles. Therefore, the use of hydro pneumatic suspension of the vehicle can keep the ride comfort in the good and poor road. This advantages are that the traditional suspension is difficult to achieved.

\section{Influence of structural parameters on stiffness characteristics}

It is can be seen from formula (6), the stiffness of the hydro pneumatic suspension system is relative to its structural parameters and the sprung mass in addition to its input displacement. The structural parameters affecting the stiffness of the hydro pneumatic suspension include the area of the A cavity, the area of the $\mathrm{C}$ cavity, the initial air volume. The basic structural parameters are the diameters of the cylinder and the piston rod associated with the area of the A cavity and the area of the $\mathrm{C}$ cavity. The influence of the diameters of the cylinder and the piston rod and the initial air volume on the stiffness of the hydro pneumatic suspension are analyzed as the following.

(1) The diameter of the piston rod

Increasing the area of the A cavity and decreasing the area of the $\mathrm{C}$ cavity have the same influence trend on the stiffness of the hydro pneumatic suspension, so it is only to analyze the influence of the diameter of the piston rod on the stiffness. Fig.3 shows the effect of the diameter of the piston rod on the stiffness of the hydro pneumatic suspension. 


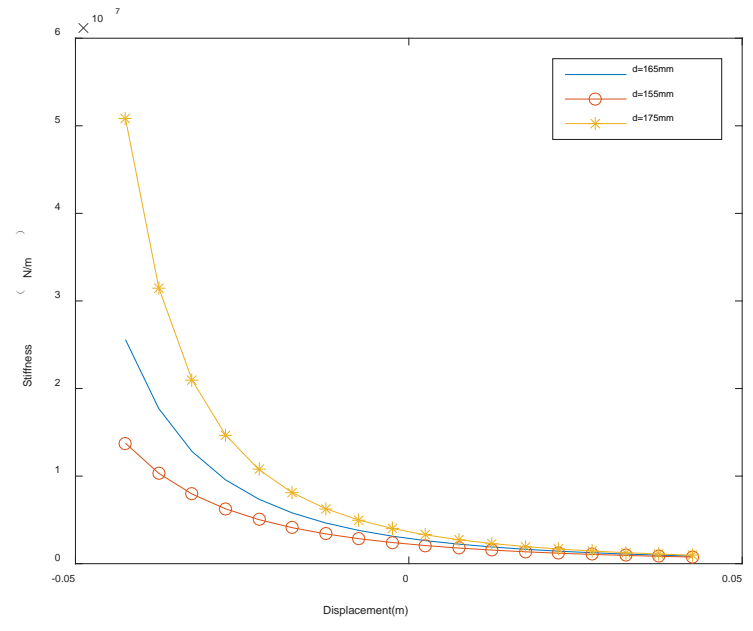

Fig. 3. The effect of the diameter of the piston rod on the stiffness

Fig. 3 shows that the stiffness of the hydro pneumatic suspension changes faster with the displacement when the diameter of the piston rod is increasing. The stiffness of the hydro pneumatic suspension is obviously reduced with the increase of the diameter of the piston rod under the same input displacement. The diameters of the cylinder and piston rod are two basic parameters of the hydro pneumatic suspension. The reasonable selection of the diameters of the cylinder and the piston rod is the basis of the design of the hydro pneumatic suspension.

(2) The initial air volume

Fig.4 shows the effect of the initial air volume on the stiffness of the hydro pneumatic suspension.

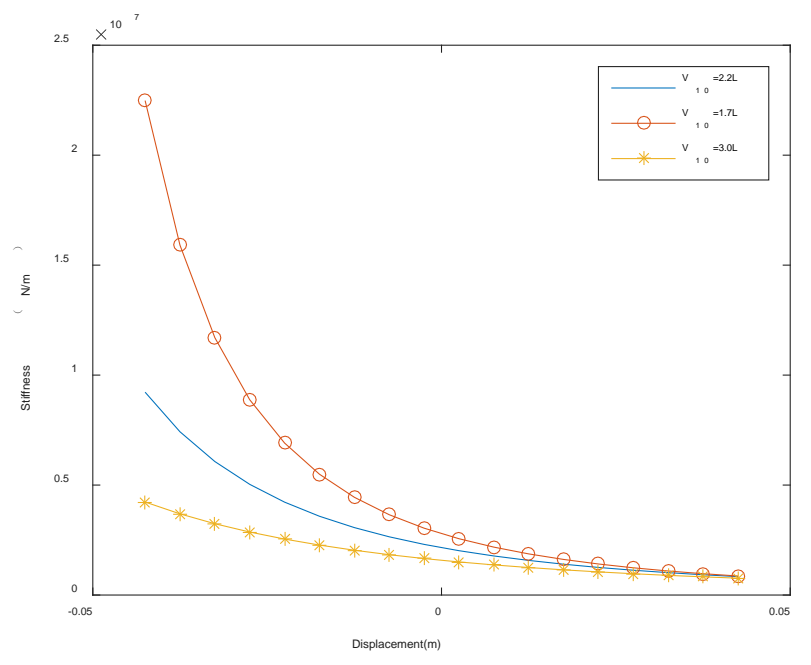

Fig. 4. The effect of initial air volume on stiffness

It can be seen from Fig.4, the stiffness of the hydro pneumatic suspension is obviously reduced with the increase of the initial air volume under the same input displacement. Therefore, it is an effective and convenient method to adjust the stiffness of the hydro pneumatic by adjusting the initial air volume after the diameters of the cylinder and the piston rod is determined. The adjustment of the initial air volume can be realized only by changing the height of the gas in the A chamber when the diameters of the cylinder and the piston rod are determined. Due to the limitation of the actual stroke of the hydro pneumatic suspension, the height of the initial gas can not be too large, and the height of the initial gas can not be too small. If the initial gas height is too small, the stiffness of the hydro pneumatic suspension is very large to result in that the inherent frequency of the spring load is high and the vehicle ride comfort is worse. Therefore, the initial air volume for hydro suspension design is very necessary. 


\section{Conclusion}

In this paper the stiffness calculation of hydro pneumatic suspension system has been completed. The influence of different structural parameter on the nonlinear stiffness characteristics of hydro-pneumatic suspension system has been simulated with MATLAB/Simulink software. The simulation results indicate that the hydro-pneumatic suspension system has the nonlinear stiffness characteristics. The dynamic stiffness is greater than the static stiffness in the same displacement, and the dynamic stiffness changes faster with the displacement. The stiffness of hydro-pneumatic suspension cylinder decreases with the increase of the initial air volume and the diameter of the piston rod.

\section{Acknowledgement}

This work was financially supported by the Fundamental Research Funds for the Central Universities(2009QJ01).

\section{References}

[1] Duan Junfa, Sun Yongsheng, Yang Zhenzhong. Dynamics simulation of hydro-pneumatic suspension based on MATLAB/SIMULINK. Tractor \& Farm Transporter, 2010,37: 59-61.

[2] Longxin Zhen, Wenming Zhang and Guobiao Wang. Review of hydro pneumatic Suspension. Nonferrous Metals(Mining), 2004, 56: 36-38.

[3] Sharp R S, Crolla D A. Road vehicle suspension design: A review. Vehicle System Dynamics, 1987,16: 167-192.

[4] Wong J R. Theory of ground vehicles. New York: John Wiley \& Sons, Inc, 2001: 236-239.

[5] Zhang Yi, Xu Maofeng, Jiang Xiaohong. Modeling, simulation and experimental research on single air chamber of oil-gas suspension. Construction Machinery and Equipment, 2004, 35(10):19-22.

[6] Feng Shi Cai. Research on nonlinear mathematical model and performance in hydro-pheumatic suspension. China Journal of Highway and Transport, 2002, 15 (3): 122-3126.

[7] Zhao Dengfeng, Wang Guoqiang. Tipper hydro pneumatic suspension mathematical model simulation . Journal of Agricultural Machinery, 2003, 34 (6): 40-43.

[8] Jinping Zhang, Experimental study and simulation on the dynamic characteristics of hydragas suspension for heavy trucks. Master degree theses of Jilin university. Changchun: Jilin university, 2003.

[9] Brian Warner, Subhash Rakheja. An analytical and experimental investigation of friction and gas spring characteristics of racing car suspension dampers. SAE 962548: 489-502.

[10] Guoqing Ma, Runhua Tan, Renzhi Wu. Non-linear mathematic model of hydro-pneumatic suspension crane vehicles and it's simulation. Chinese Journal of Mechanical Engineering, 2002,38(5): 95-99. 